\title{
riccafd
}

Revista Iberoamericana de Ciencias de la Actividad Física y el Deporte

\section{ALTERNATIVAS A LA METODOLOGÍA TRADICIONAL PARA COMBATIR LA OBESIDAD EN ESCOLARES: ENTRENAMIENTO CONCURRENTE POLARIZADO}

\section{ALTERNATIVES TO TRADITIONAL METHODOLOGY TO COMBAT OBESITY IN SCHOOLCHILDREN: POLARIZED CONCURRENT TRAINING}

Morente-Oria, $\mathrm{H}$.

Facultad de Ciencias de la Educación. Universidad de Málaga. España.

Correspondencia: Honorato Morente Oria. hono@uma.es

Código UNESCO: 3212, Salud Pública.

Clasificación Consejo Europa: 17. Otras: Actividad Física y Salud.

Recibido el 16 de abril de 2019.

Aceptado el 26 de septiembre de 2019.

DOI: http://dx.doi.org/10.24310/ 10.24310/riccafd.2019.v8i3.7489.

\section{RESUMEN}

Con este trabajo queremos poner de manifiesto otro tipo de metodología distinta a las que se realizan tradicionalmente desde las organizaciones más influyentes (ACSM-American College Sport Medicine, WHO-World Health Organization y AHA-American Heart Association) para luchar contra un problema incipiente en la escuela de hoy como es el sobre peso y la obesidad.

El entrenamiento concurrente (fuerza-resistencia) unido con un trabajo de intensidad polarizada (alta y baja) produce mejores resultados para la reducción de la obesidad que el trabajo aislado de ambas y de intensidades submáximas como se venía trabajando en los protocolos más clásicos.

Palabras clave: entrenamiento concurrente, HIIT, entrenamiento de fuerza, obesidad, diabetes.

\section{ABSTRACT}

With this work we want to highlight another type of methodology different from those traditionally carried out by the most influential organizations (ACSMAmerican College Sport Medicine, WHO-World Health Organization and AHAAmerican Heart Association) to fight against an incipient problem in school today such as overweight and obesity. 
The concurrent training (strength-resistance) combined with a work of polarized intensity (high and low) produces better results for the obesity reduction than the isolated work of both and submaximal intensities as it had been working in the most classic protocols.

Key words: concurrent training, HIIT, strength training, obesity, diabetes.

\section{INTRODUCCIÓN}

La actividad física (AF) es un instrumento ideal para mejorar aspectos de salud y combatir el sedentarismo tan acuciante en nuestros días, también se utiliza para prevenir y tratar la obesidad y todas las enfermedades consecuentes de esta actividad como pudiera ser la diabetes en sus distintas formas. Según los datos de la Organización Mundial para la Salud (WHO-World Health Organization por sus siglas en inglés) en su último informe de 2016 revela un dato muy preocupante y es que desde 1980 la obesidad se ha duplicado, donde, a 2014, un 39\% de las personas adultas tenían sobrepeso y un $13 \%$ eran obesas ${ }^{1}$. Las recomendaciones generales que aporta la OMS para el problema de la obesidad y la prevención de enfermedades crónicas no contagiosas (ECNC) son en dos líneas, por un lado, el control de la alimentación, y la realización de AF; pero las recomendaciones que hacen sobre esta última son muy genéricas y en ocasiones no optimizado para el control y la mejora de factores de riesgo como la obesidad y la diabetes ${ }^{2}$.

Cuando nuestra misión es la mejora de algunas patologías, en nuestro caso de obesidad y la diabetes, lo primero que debemos conocer son los parámetros de entrenamiento que marcan las distintas líneas de trabajo para poder optimizar el trabajo y conseguir los mejores resultados con los medios disponibles. En cuanto a medios disponibles en la mayoría de los casos de obesidad, la mayor limitación suele ser el tiempo de trabajo y también lo abordaremos en este texto.

Los parámetros más importantes para la periodización de los entrenamientos son el volumen, la intensidad, la frecuencia y el tipo de trabajo realizado (generalmente resistencia y fuerza). De estos parámetros vamos a tener en cuenta en este trabajo el tipo de trabajo y la intensidad del ejercicio sin menospreciar que hay que tener también los otros aspectos en cuenta.

En un análisis de las recomendaciones tradicionales que desde las organizaciones más influyentes (ACSM-American Collegue Sport Medicine, WHO-World Health Organization y AHA-American Heart Association) hacen de AF para la salud, como veremos en la revisión, nos parecen insuficientes a la luz de las nuevas corrientes de entrenamiento y los estudios recientes sobre entrenamiento y salud ${ }^{3-9}$.

\section{SITUACIÓN ACTUAL}

Cuando hablamos de recomendaciones de AF enfocadas en la salud, resulta muy complicado hablar de generalidades ya que depende de multitud de factores como pueden ser la experiencia previa, patologías asociadas, lesiones osteoarticulares, nivel actual de condición física, objetivo de la persona en función de su morfología y situación actual, etc. Todo ello hace muy complicado generalizar 
el tipo de AF a realizar por lo que la mayoría de organizaciones lo hacen para la población general presumiendo estar libre de patologías.

Las organizaciones de las que hemos hablado en la introducción vienen realizando, desde los años 60, recomendaciones de actividad física para una vida más saludable. Actualmente las recomendaciones de estas organizaciones están enfocadas en distintos aspectos y toman como medidas distintos aspectos.

En el caso de la ACSM centra sus objetivos de entrenamiento en 4 aspectos fundamentales; ejercicio cardiorespiratorio; entrenamiento de fuerza; flexibilidad; ejercicio neuromotor ${ }^{10}$.

Por otro lado, la WHO realiza una recomendación de AF por franja de edad; de 5 a17 años; de 18 a 64 años; de 65 años en adelante. En el primer grupo de edad sólo hacen referencia al tiempo que deben realizar actividad física a la semana (60 min/día AF de moderada a vigorosa); en el segundo grupo nos habla del trabajo cardiovascular (150 min/semana de moderada a vigorosa) pero también hablan de trabajo de fuerza (2 o más días/semana); en el último grupo también nos hacen referencia al trabajo cardiovascular (150 min/semana moderado o 75 min/semana vigoroso) y trabajo de fuerza adaptado (2 o más días/semana) ${ }^{11}$.

En lo referente a la AHA (asociación americana del corazón) hace una clasificación de las recomendaciones en función de: salud cardiovascular general (30 min 5 días/semana AF moderada o 25 min 3días/semana actividad vigorosa y 2 días/ semana de trabajo de fuerza de moderada a alta intensidad) y reducción de tensión arterial y el colesterol (40 min de 3 a 4 días/semana AF de moderada a vigorosa) ${ }^{12}$.

Estas organizaciones han incluido en los últimos años el trabajo de fuerza (Resistance Training en inglés) como reconocimiento a los estudios más actuales en el entrenamiento y las ventajas que aporta el trabajo muscular al estado de bienestar y salud. Sin embargo, no realizan aportaciones muy concretas de cómo debería ser ese trabajo de fuera y como debe ir incluido con el resto de carga de trabajo a lo largo de la semana sólo la ACSM en 2009 realizó una progresión de las cargas de fuerza ${ }^{13}$ y en 2011 incluyó el trabajo de flexibilidad ${ }^{14}$.

Analizando los modelos clásicos Vs nuevas corrientes como los entrenamientos concurrentes (en nuestro caso trabajo de fuerza y resistencia en la misma sesión de entrenamiento) marcan una serie de diferencias que veremos a continuación.

Por un lado, la intensidad del ejercicio. Mientras en los modelos clásicos la intensidad del ejercicio estaba en zona II cerca del umbral anaeróbico (70-90 VO2 max y $85-90 \% \mathrm{FC}_{\max }$ ) en los modelos polarizados las zonas de trabajo estarían en Zona I y Zona III, por debajo del umbral aeróbico (50-60 VO2max y 65-70\% FCmax) o por encima del umbral anaeróbico $\left(70-90 \mathrm{VO}_{2 \max } \text { y 85-90\% } \mathrm{FC}_{\max }\right)^{15-16}$.

\begin{tabular}{|c|c|c|}
\multicolumn{1}{c|}{$50-60 \mathrm{VO}_{2 \max }$ y $65-70 \% \mathrm{FC}_{\max }$} & Umbral anaeróbico \\
\hline Umbral & aeróbico & Zona III \\
\hline Zona I & Zona II & Zon \\
\hline
\end{tabular}


Este tipo de entrenamientos denominados polarizados, en los que no se trabaja en zona II, favorecen el trabajo de movilización de grasas no sólo durante el ejercicio sino también en el post ejercicio (EPOC- Excess Post-exercise Oxygen Consumption) a pesar de creencias, muy arraigadas, donde la mejor forma de oxidar grasas se cree que es con un trabajo moderado $\left(60-70 \% \mathrm{FC}_{\text {max }}\right)$ de más de $30 \mathrm{~min}$. Es importante dentro de las vías oxidativas el EPOC ya que ayuda a movilizar hasta 48 horas después ${ }^{17}$.

En los modelos clásicos existía la creencia de que los entrenamientos de resistencia y de fuerza eran incompatibles ya que consideraban sus capacidades antagónicas y lo que se denominó el proceso de la interferencia ${ }^{18}$. Numerosos estudios han dejado de manifiesto que existen mejoras en el entrenamiento de ambas capacidades, pero en algunos estudios argumentan que tiene mejores resultados realizar el trabajo de fuerza antes del trabajo de resistencia, sobre todo si las cargas de fuerza son muy elevadas aunque no pueden asegurar que no exista interferencia entre ambos trabajos ${ }^{19}$.

Si nos centramos a nivel de los procesos bioquímicos que producen el tejido adiposo y en especial a la leptina, que es considerada el regulador de la producción de este en el organismo y que controla el peso corporal, encontramos distintos estudios que concluyen que el ejercicio físico influye positivamente en el control de los niveles de leptina ${ }^{20-22}$.

Pero si analizamos un poco más encontramos diversos estudios que van un paso más allá, indicando que el entrenamiento de alta intensidad o en los entrenamientos con entrenamientos que causa un mayor nivel de estrés en el organismo, son más eficientes para controlar el nivel de leptina en el organis$\mathrm{mo}^{23}$.

Otros estudios como los de Rosa et al y Zafeiridis et al. profundizan un poco más en las posibilidades de movilización de niveles de Leptina y nos hablan de entrenamiento concurrente (Entrenamiento que alterna en la misma sesión entrenamientos cardiovasculares con entrenamientos contraresistencia)concurrente y concluyen que este reduce los niveles de leptina en el organismo, pero diferencian entre los niveles de intensidad, siendo estas diferencias, estadísticamente significativas, sólo en los entrenamientos concurrentes cuando se utiliza alta intensidad en sus protocolos de entrenamiento ${ }^{24-25}$.

Cuando nos referimos al entrenamiento concurrente podríamos estar pensando en trabajo con personas sanas, pero existe evidencia científica que sugiere que este tipo de trabajo mejora desde personas no entrenadas sin patologías hasta personas con patologías cardiacas crónicas ya que mejora la fuerza muscular, la capacidad submáxima y la calidad de vida con respecto al entrenamiento de resistencia aislado ${ }^{26-27}$.

Estamos viendo numerosos ejemplos de evidencias científicas altas de la mejora del entrenamiento concurrente con respecto a entrenamientos de fuerza y resistencia de forma aislada pero aún no está claro cuál es el estímulo óptimo para obtener los mayores beneficios ${ }^{28-30}$. 


\section{CONCLUSIÓN}

Una cosa está clara y es que a pesar de los intentos de las organizaciones más importantes por realizar recomendaciones de trabajo para la salud aún no está claro cuáles son las pautas a seguir en cuanto a ejercicios y estímulos para obtener los mejores resultados y optimizar los entrenamientos. Lo que si queda claro es que el entrenamiento concurrente y con intensidades polarizadas (altas y bajas) son herramientas más útiles para la lucha contra la obesidad que el trabajo aislado de fuerza o resistencia.

Si a eso unimos el problema de la falta de tiempo que padece la sociedad actual consideramos que este tipo de entrenamiento puede ser más optimizado que otros tipos de entrenamientos de corte más clásico.

Esta revisión sugiere la profundización en los diferentes modos de trabajo concurrente y estudiar las distintas intensidades y frecuencias para intentar ver que trabajo es el más optimizado para la mejora de la salud en personas obesas y diabéticas.

\section{BIBLIOGRAFÍA}

1. WHO: World Health Organization [Internet]. Ginebra: WHO; junio 2016 [citado 20 may 2017]. Página WHO (World Health Organization). Disponible en: http:// www.who.int/mediacentre/factsheets/fs311/en/

2. Varela Sanz A. Efectos del entrenamiento concurrente, polarizado y tradicio- nal, sobre la condición física saludable [tesis doctoral en Internet]. La Coruña:

Universidade da Coruña; 2014 [citada 24 may 2017]. 280p. Disponible en: http:// ruc.udc.es/dspace/handle/2183/12463

3. Sheikholeslami-Vatani $D$, et al. The effect of concurrent training order on hormonal responses and body composition in obese men. Science \& Sports, Diciembre de 2015;30(6):335-341.

4. Mann S, Beedie C, Jimenez A. Differential effects of aerobic exercise, resistance training and combined exercise modalities on cholesterol and the lipid profile: review, synthesis and recommendations. Sports Med AuckI NZ. febrero de 2014;44(2):211-21.

5. Taipale RS, Mikkola J, Vesterinen V, Nummela A, Häkkinen K. Neuromuscular adaptations during combined strength and endurance training in endurance runners: maximal versus explosive strength training or a mix of both. Eur J Appl Physiol. febrero de 2013;113(2):325-35.

6. Boullosa DA, Abreu L, Tonello L, Hofmann P, Leicht AS. Exercise is medicine: case report of a woman with smoldering multiple myeloma. Med Sci Sports Exerc. julio de 2013;45(7):1223-8.

7. Mikkola J, Rusko H, Izquierdo M, Gorostiaga EM, Häkkinen K. Neuromuscular and cardiovascular adaptations during concurrent strength and endurance training in untrained men. Int J Sports Med. septiembre de 2012;33(9):702-10.

8. Izquierdo M, Häkkinen K, Ibáñez J, Kraemer WJ, Gorostiaga EM. Effects of combined resistance and cardiovascular training on strength, power, muscle cross-sec- 
tional area, and endurance markers in middle-aged men. Eur J Appl Physiol. mayo de 2005;94(1-2):70-5.

9. Sillanpää E, Laaksonen DE, Häkkinen A, Karavirta L, Jensen B, Kraemer WJ, etal. Body composition, fitness, and metabolic health during strength and endurance training and their combination in middle-aged and older women. Eur J Appl Physiol. mayo de 2009;106(2):285-96.

10. ACSM: American College of Sports Medicine [Internet]. Indianapolis: ACSM; agosto 2011. [citado 25 may 2018]. Página ACSM (American College of Sports Medicine). Disponible en: http://www.acsm.org/about-acsm/media-room/newsreleases/2011/08/01/acsm-issues-new-recommendations-on-quantity-and-quali- tyof-exercise

11. WHO: World Health Organization [Internet]. Ginebra: WHO; 2011 [citado 25 may 2017]. Página WHO (World Health Organization). Disponible en: http://www. who.int/dietphysicalactivity/factsheet_recommendations/en/

12. AHA: American Heart Association [Internet]. Ginebra: AHA; julio 2016 [citado 25 may 2018]. Página AHA (American Heart Association). Disponible en: http://www.heart.org/HEARTORG/HealthyLiving/PhysicalActivity/FitnessBasics/ American-Heart-Association-Recommendations-for-Physical-Activity-in-Adults_ UCM_307976_Article.jsp\#.WSnE2Illryu4

13. Ratamess NA, Alvar BA, Evetoch TK, Housh TJ, Kibler WB, Kraemer WJ, et al. American College of Sports Medicine position stand. Progression models in resistance training for healthy adults. Med Sci Sports Exerc. marzo de 2009; 41(3):687-708.

14. Garber CE, Blissmer B, Deschenes MR, Franklin BA, Lamonte MJ, Lee I-M, et al. American College of Sports Medicine position stand. Quantity and quality of exercise for developing and maintaining cardiorespiratory, musculoskeletal, and neuromotor fitness in apparently healthy adults: guidance for prescribing exercise. Med Sci Sports Exerc. julio de 2011; 43(7):1334-59.

15. Boullosa DA, Nakamura FY, Ruiz JR. Effectiveness of polarized training for rowing performance. Int J Sports Physiol Perform. diciembre de 2010;5(4):431- 432; 432-436.

16. Esteve-Lanao J, Foster C, Seiler S, Lucia A. Impact of training intensity distribution on performance in endurance athletes. J Strength Cond Res Natl Strength Cond Assoc. diciembre de 2007;21(3):943-9.

17. Heredia JR, Isidro F, Chulvi I, Roig J, Moral S, Molins A. Sobrepeso/obesidad, ejercicio físico y salud: intervención mediante programas de fitness. Barcelona: Wanceulen; 2008

18. Docherty D, Sporer B. A proposed model for examining the interference phenomenon between concurrent aerobic and strength training. Sports Med Auckl NZ. diciembre de 2000;30(6):385-94.

19. Jones $T$ W, Howatson G, Russell M, French DN. Effects of strength and endurance exercise order on endocrine responses to concurrent training. European journal of sport science, Junio 2017;17(3):326-334.

20. Rosa G, Cruz I, Mello DBd, Fortes MdSR, Dantas EHM. Plasma levels of leptin in overweight adults undergoing concurrent training International SportMed Journal $2010 ; 11(3)$. 
21. Eguchi R, Cheik NC, Oyama LM, Nascimento CMOd, Mello MTd, Tufik S, et al. Efeitos do exercício crônico sobre a concentração circulante da leptina e grelina em ratos com obesidade induzida por dieta. Rev Bras Med Esporte 2008;14:182-187.

22. Negrão AB, Licinio J. Leptina: o Diálogo entre Adipócitos e Neurônios. Arq Bras Endocrinol Metab 2000;44(3):205-214.

23. Hulver MW. Plasma leptin and exercise: recent findings. Sports Med2003; 33(7):473-482.

24. Rosa G, Braga D, Daoud R, Cruz I, Dantas HM. Concentración de Leptina en adultos con sobrepeso sujetos a un entrenamiento concurrente. Mot. Hum., 10(2): 95-102, 2010.

25. Zafeiridis A, Smilios I, Considine R \& Tokmakidis S. Serum leptin responses after acute resistance exercise protocols. J Appl Physiol2003;94:591-597.

26. Gary RA, Cress ME, Higgins MK, Smith AL, Dunbar SB. Combined aerobic and resistance exercise program improves task performance in patients with heart failure. Arch Phys Med Rehabil. septiembre de 2011;92(9):1371-81.

27. Beckers PJ, Denollet J, Possemiers NM, Wuyts FL, Vrints CJ, Conraads VM. Combined endurance-resistance training vs. endurance training in patients with chronic heart failure: a prospective randomized study. Eur Heart J. agosto de 2008;29(15):185866.

28. Mann S, Beedie C, Jimenez A. Differential effects of aerobic exercise, resistance training and combined exercise modalities on cholesterol and the lipid profile: review, synthesis and recommendations. Sports Med AuckI NZ. febrero de 2014;44(2):211-21.

29. Taipale RS, Mikkola J, Vesterinen V, Nummela A, Häkkinen K. Neuromuscular adaptations during combined strength and endurance training in endurance runners: maximal versus explosive strength training or a mix of both. Eur J Appl Physiol. febrero de 2013;113(2):325-35.

30. Mikkola J, Rusko H, Izquierdo M, Gorostiaga EM, Häkkinen K. Neuromuscular and cardiovascular adaptations during concurrent strength and endurance training in untrained men. Int J Sports Med. septiembre de 2012;33(9):702-10. 